\title{
Coupled ptychography and tomography reconstruction of experimental data
}

\author{
Maik Kahnt ${ }^{1,2, \uparrow_{*}}$, Johannes Becher ${ }^{3}$, Dennis Brückner ${ }^{1,2}$, Yakub Fam ${ }^{3, \ddagger}$, Thomas Sheppard ${ }^{3,4}$, Tobias \\ Weissenberger ${ }^{5}$, Felix Wittwer ${ }^{1,2}$, Jan-Dierk Grunwaldt ${ }^{3,4}$, Wilhelm Schwieger ${ }^{5}$ and Christian G. Schroer ${ }^{1,2}$ \\ ${ }^{1}$ Deutsches Elektronen-Synchrotron DESY, Notkestraße 85, 22607 Hamburg, Germany \\ ${ }^{2}$ Department Physik, Universität Hamburg, Luruper Chaussee 149, 22761 Hamburg, Germany \\ ${ }^{3}$ Institute for Chemical Technology and Polymer Chemistry, Karlsruhe Institute of Technology, Engesserstr. 20, 76131 Karlsruhe, \\ Germany \\ ${ }^{4}$ Institute of Catalysis Research and Technology, Karlsruhe Institute of Technology, Hermann-von-Helmholtz Platz 1,76344 \\ Eggenstein-Leopoldshafen, Germany \\ ${ }^{5}$ Institute of Chemical Reaction Engineering, Friedrich-Alexander-University Erlangen-Nürnberg, Egerlandstraße 3, 91058 \\ Erlangen, Germany \\ †now MAX IV Laboratory, Lund University, Fotongatan 2, 22594 Lund, Sweden \\ ${ }^{\ddagger}$ now Thermo Fisher Scientific, Vlastimila Pecha 1282/12, 62700 Brno, Czech Republic
}

maik.kahnt@maxiv.lu.se

Three-dimensional X-ray microscopy by ptychographic tomography is usually per formed by separating the steps of acquiring twodimensional ptychographic reconstructed projection images at different projection angles and afterwards performing the threedimensional tomographic reconstruction. Recently it has been suggested that those two separate steps can be coupled / joined together, allowing for the sharing of information between angular views during the ptychographic reconstruction step [1, 2, 3]. We performed such a coupled X-ray ptychographic tomography reconstruction for the first time on an experimental dataset, improving the achieved resolution in the process [4]. Furthermore we validated the predicted relaxation of the overlap criterion between adjacent scan positions in the tomographic plane by successively leaving out columns of recorded diffraction patterns and achieving robust reconstructions even beyond the point of no overlap between neighboring scan points.

[1] D. Gürsoy, "Direct coupling of tomography and ptychography," Opt. Lett., vol. 42, pp. 3169-3172, Aug 2017.

[2] T. Ramos, B. E. Grønager, M. S. Andersen, and J. W. Andreasen, "Direct three- dimensional tomographic reconstruction and phase retrieval of far-field coherent diffraction patterns," Phys. Rev. A, vol. 99, p. 023801, Feb 2019.

[3] S. Aslan, V. Nikitin, D. J. Ching, T. Bicer, S. Leyffer, and D. Gürsoy, "Joint ptycho-tomography reconstruction through alternating direction method of multipliers," Opt. Express, vol. 27, pp. 9128-9143, Mar 2019.

[4] M. Kahnt, J. Becher, D. Brückner, Y. Fam, T. Sheppard, T. Weissenberger, F. Wittwer, J.-D. Grunwaldt, W. Schwieger, and C. G. Schroer, "Coupled ptychography and tomography algorithm improves reconstruction of experimental data," Optica, vol. 6, pp. 1282-1289, Oct 2019.

Keywords: Ptychography, Tomography 